\title{
Uso del programa simapro para evaluar coagulantes naturales en el tratamiento de aguas
}

\author{
Use of the simapro program to evaluate natural coagulants in water treatment
}

\author{
Leticia Angélica Flores Pérez ${ }^{1 *}$, Rubén Lostado Lorza ${ }^{2}$, Marina Corral Bobadilla ${ }^{2}$ \\ Benemérita Universidad Autónoma de Puebla, Facultad de Ingeniería Química. México. \\ Edificio Departamental de la Facultad de Ingeniería Industrial. Logroño, España.
}

\section{RESUMEN}

El Análisis del Ciclo de Vida (ACV) es uno de los modelos cuantitativos de mayor uso en las empresas para la evaluación del impacto ambiental de diversos procesos industriales. El presente trabajo consistió en realizar el ACV del proceso de coagulación-floculación en aguas residuales mediante el uso del programa SimaPro, en la que se analizaron 195 datos generados del proceso de tratamiento de aguas residuales con distintos coagulantes (Ecotan Bio 90D, 100 y G-150) y diferentes parámetros del proceso: dosis de coagulante 1 a $6 \mathrm{~mL}$, velocidad de agitación de 50 a 100 rpm, tiempo de agitación de 30 a 120 s y temperatura de tratamiento de 10 a $20{ }^{\circ} \mathrm{C}$. Los resultados del programa SimaPro muestran un impacto ambiental prácticamente nulo con el Ecotan Bio 90D y 100 en la generación de Gases de Efecto Invernadero, acidificación/eutrofización, uso de suelo y recursos extraíbles.

Palabras clave: coagulación-floculación, ACV, SimaPro, impacto ambiental.

\section{ABSTRACT}

Life Cycle Analysis (LCA) is one of the most used quantitative models in companies for the evaluation of the environmental impact of various industrial processes. The present work consisted in performing the coagulation-flocculation process ACV in wastewater by using the SimaPro program. For this, 195 data generated from the wastewater treatment process with different coagulants were analyzed (Ecotan Bio 90D, 100 and G -150), considering different process parameters: coagulant dose 1 to $6 \mathrm{~mL}$, agitation speed from 50 to $100 \mathrm{rpm}$, agitation time from 30 to $120 \mathrm{~s}$ and treatment temperature from 10 to $20^{\circ} \mathrm{C}$. The SimaPro program results show a practically zero environmental impact with the Ecotan Bio $90 \mathrm{D}$ and 100 in the generation of greenhouse gases, acidification / eutrophication, land use and extractable resources.

Key words: coagulation-flocculation, ACV, SimaPro, environmental impact.

\section{INTRODUCCIÓN}

Diferentes instituciones gubernamentales y no gubernamentales de todos los países han desarrollado iniciativas enfocadas a la implementación del Análisis del Ciclo de Vida (ACV) en los procesos industriales y en los productos, con la finalidad de generar conciencia ambiental en las empresas y

\section{Volumen XXI, Número 3}

así, establecer el cumplimiento de los estándares de la gestión ambiental y la aplicación de las 3R (Reducir, Reutilizar y Reciclar) (Heijungs et al., 2010), para minimizar la contaminación ambiental y por ende generar una sostenibilidad ambiental que implique la producción de productos reciclables de un 95 \% y la reducción del costo del ciclo de vida en cada una de las etapas del proceso de un producto, así como, la reducción del daño ambiental en los recursos naturales (Roche, 2004; Seow et al., 2016). Por ello, en el desarrollo del ACV surge la necesidad de realizar una evaluación ambiental en los procesos industriales, en especial en el tratamiento de aguas residuales, ya que todavía no se cuenta con la información suficiente para llevar a cabo un ACV de los procesos (Manuilova et al., 2009; Seow et al., 2016). La evaluación ambiental tiene que estar basada desde los sistemas de producción, la implementación de nuevos procesos en el tratamiento de aguas residuales, empleo de nuevos productos amigables con el ambiente, etc. además de integrar el uso de metodologías ligadas con modelos cuantitativos y de criterios de tipo político, económico, social y ambiental para la toma de decisiones que conciernen la mejora continua de los procesos (Höjer et al., 2008).

Durante el desarrollo del ACV se debe de definir correctamente los factores unitarios y los límites del sistema de cada una de las etapas del proceso con la finalidad de optimizar el proceso, implementando nuevas metodologías para la gestión adecuada de los recursos energéticos que disminuyan costos y la huella del carbono (Rodríguez et al., 2011; Lorenzo et al., 2015, 2016a, 2016b; Singh et al., 2016).

Dentro de los modelos empleados para el ACV de los procesos industriales se encuentran los europeos IMPACT 2002+ y EDIP2003; japonés LIME; estadounidenses TRACI y LSCEA; y el canadiense LUCAS. En estos estudios se ha empleado el SimaPro como una de las herramientas de elevada potencia por su grado de impacto en los procesos industriales y en el Ecodiseño, además por su fácil manejo e interpretación para los usuarios, ya que es una fuente que engloba una amplia base de datos concernientes al estudio de impacto ambiental, realiza comparaciones con otros procesos industriales para la optimización y la toma de decisiones con la finalidad de proporcionar una mejora continua ambiental en sus procesos y productos desde la "cuna a la tumba" (Seow et al., 2016; Manuilova et al., 2009).

Además, de acuerdo a los ensayos reportados por diversas fuentes bibliográficas muestran que el proceso re-

*Autor para correspondencia: Leticia Angélica Flores Pérez Correo electrónico: angyflo2008@hotmail.com

Recibido: 24 de enero de 2019 Aceptado: 19 de marzo de 2019 
sulta ser afectado de forma significativa por cada uno de los siguientes parámetros: tipo y origen de agua a tratar, tipo y naturaleza del coagulante (natural o sintético), concentración del coagulante/floculante, tiempo y velocidad de agitación y el tiempo de retención, teniendo un impacto crucial tanto en el proceso como en el entorno ambiental (loannou et al., 2015).

El proceso de coagulación se produce por la existencia de partículas suspendidas en el agua a un pH neutro, cuya carga superficial es negativa y los iones positivos que están en la disolución son atraídos y absorbidos hacia las partículas aniónicas, de aquí se puede observar cuatro zonas (capa superficial, capa de Stern, capa difusa de Gouy-Chapman y la capa doble) que determinan el comportamiento de los iones en el proceso. Por otro lado, el proceso de floculación es el paso subsecuente de la coagulación y se basa en promover el contacto entre las partículas coloidales para formar aglomerados por la adsorción de polímeros de alto peso molecular o de cadenas largas, provocando de esta manera la eliminación del agregado de la fase acuosa, teniendo tres tipos de floculación de acuerdo al modo de acción del agente iónico, siendo la más importante la floculación "bridging", ya que es donde ocurre la adsorción simultánea de partículas a bajas dosis de floculante. En todo caso, los dos procesos antes mencionados se rigen fundamentalmente por fuerzas electrostáticas y de atracción.

Además, es de destacar que actualmente se están sustituyendo los coagulantes/floculantes de iones multivalentes $\left(\mathrm{Al}^{3+} \mathrm{Fe}^{3+}, \mathrm{Fe}^{2+}, \mathrm{Ca}^{2+} \circ \mathrm{Mg}^{2+}\right)$ por coagulantes/floculantes de origen natural (Opuntia spp., quitosan, Moringa Oleifera, etc.) que son más amigables con el ambiente y han mostrado tener la misma efectividad en la clarificación, eliminación de la Demanda Biológica de Oxígeno (DBO) y fosfatos (Oladoja, 2015; Dorca et al., 2017; Mateus et al., 2017; Rebah y Siddeeg, 2017; Saritha et al., 2017).

Por ende, el objetivo de este trabajo se centró en realizar una evaluación ambiental del proceso de coagulaciónfloculación en el tratamiento de aguas residuales, a través de la aplicación de la metodología del ACV con el programa SimaPro.

\section{MATERIALES Y MÉTODOS}

\section{Muestreo, caracterización y tratamiento fisicoquímico de agua residual}

El muestreo, la caracterización y el tratamiento fisicoquímico de agua residual fueron realizados por Yanguas (2017).
Muestreo de agua residual. Las muestras de agua residual proceden de la Estación Depuradora de Aguas Residuales de Logroño (EDAR), ubicada en las coordenadas geográficas $42^{\circ} 27^{\prime} 56.33^{\prime \prime}$ Norte y $2^{\circ} 25^{\prime} 00.20^{\prime \prime}$ Oeste y se tomaron después de la etapa de desarenado-desengrasado en enero de 2017.

Caracterización de agua residual. Las muestras fueron caracterizadas en la EDAR y los parámetros de caudal, $\mathrm{pH}$, sólidos en suspensión, demanda química de oxígeno, demanda biológica de oxígeno, nitrógeno total, amoniaco y fósforo total están reportados en la página de La Rioja (www. larioja.org), las cuales fueron empleadas para el desarrollo del diseño experimental (Tabla 1).

Tratamiento fisicoquímico. En el tratamiento fisicoquímico se emplearon tres tipos de coagulante natural de la serie Ecotan Bio (90D, 100, G-150), proporcionados por la empresa servyeco los cuales se caracterizan por poseer una estructura polimérica de polifenoles, estas pueden actuar como coagulante/floculante o auxiliares del proceso de coagulación-floculación, no modifican la conductividad del agua tratada, se pueden emplear como agentes antimicrobianos para disminuir la población de microorganismos patógenos (www.servyeco.com). Para el tratamiento, Yanguas (2017) realizó primero el diseño experimental que consistió en el uso del Diseño de Composición Central (CCD) para el ajuste de modelos de segundo orden, generando de esta manera 195 ensayos divididos en 54 ensayos de entrenamiento y 11 de testeo, en cada ensayo varió los parámetros de entrada del sistema: velocidad de agitación (50-100 rpm), tiempo de agitación (30-120 s), dosis de coagulante (1-6 mL), temperatura $\left(10-20^{\circ} \mathrm{C}\right)$, turbidez (40-140 NTU). Finalmente, cada uno de los parámetros generados por el CCD se introdujeron en el equipo de prueba de jarras (JAR TEST FLOCUMATIC marca SELECTA) para el tratamiento de aguas residuales.

\section{Determinación de los límites del sistema e inventario de datos para el ACV}

Los límites del sistema del proceso de coagulaciónfloculación se determinaron a partir de los parámetros de entrada, estableciendo como flujos de entrada de materiales y energía: el volumen de agua convertido en unidades de masa $(\mathrm{kg})$, velocidad de agitación y temperatura en unidades de energía (kWh) y la turbidez (NTU); como flujos de salida fueron considerados los subproductos generados en el proceso: turbidez final (NTU).

Tabla 1. Caracterización del agua residual sin tratamiento con fecha de muestreo 01/2017.

Table 1. Characterization of wastewater without treatment with sampling date 01/2017.

\begin{tabular}{lcccccccc}
\hline $\mathbf{Q}\left(\mathbf{m}^{3} / \mathbf{d}\right)$ & Eq.hab & $\mathbf{p H}$ & $\mathbf{S S}_{\mathrm{i}}(\mathbf{m g} / \mathbf{L})$ & $\mathrm{DQO}(\mathbf{m g} / \mathbf{L})$ & $\mathbf{D B O}(\mathbf{m g} / \mathbf{L})$ & $\begin{array}{c}\mathbf{N}_{\mathrm{T}} \\
(\mathbf{m g} / \mathbf{L})\end{array}$ & $\begin{array}{c}\mathbf{N H}_{4}^{+} \\
(\mathbf{m g} / \mathbf{L})\end{array}$ & $\mathbf{P}_{\mathrm{T}}(\mathbf{m g} / \mathbf{L})$ \\
\hline 4023801 & 483445.67 & 7.54 & 190.82 & 320.53 & 151.45 & 37.84 & 22.84 & 4.35 \\
\hline
\end{tabular}

Q: Caudal medio diario; Eq.hab: Tratamiento de vertidos equivalente al número de habitantes; SS: Sólidos en suspensión inicial, DQO:

Demanda Química de Oxígeno; $\mathrm{N}_{\mathrm{T}}$ : Nitrógeno total; $\mathrm{P}_{\mathrm{T}}$ : Fósforo total. 


\section{Herramienta informática para el ACV}

Para el estudio del ACV en el proceso de coagulaciónfloculación se empleó el programa SimaPro versión 8.4.0. Con los datos obtenidos del tratamiento fisicoquímico se alimentó el programa SimaPro, desarrollando diversos modelos comparativos con los distintos coagulantes empleados y la turbidez inicial (Figura 1), para obtener los datos de impacto ambiental caracterizadas en las cuatro categorías listadas del programa: efectos derivados del cambio climático, acidificación/eutrofización, en el suelo por el uso de la biomasa (co-producto del tratamiento) y el efecto derivado de los recursos fósiles/minerales extraídos.

\section{RESULTADOS Y DISCUSIÓN}

Eficiencia del proceso de coagulación-floculación con tres tipos de coagulante natural

Los mejores resultados de la reducción de la turbidez de aguas residuales brutas con 140 NTU aplicando el proceso de coagulación-floculación y haciendo uso de tres coagulantes naturales de la serie Ecotan Bio, muestran una tasa de reducción de la turbidez de hasta un $92 \%$ con el coagulante Ecotan Bio G150 y de un 95 \% con los coagulantes Ecotan Bio $90 \mathrm{D}$ y 100 , cuando se aplica una mínima dosis de coagulante (1 mL) y una baja energía de agitación (1254.73 kWh) (ver extracto de resultados Tabla 2 ).

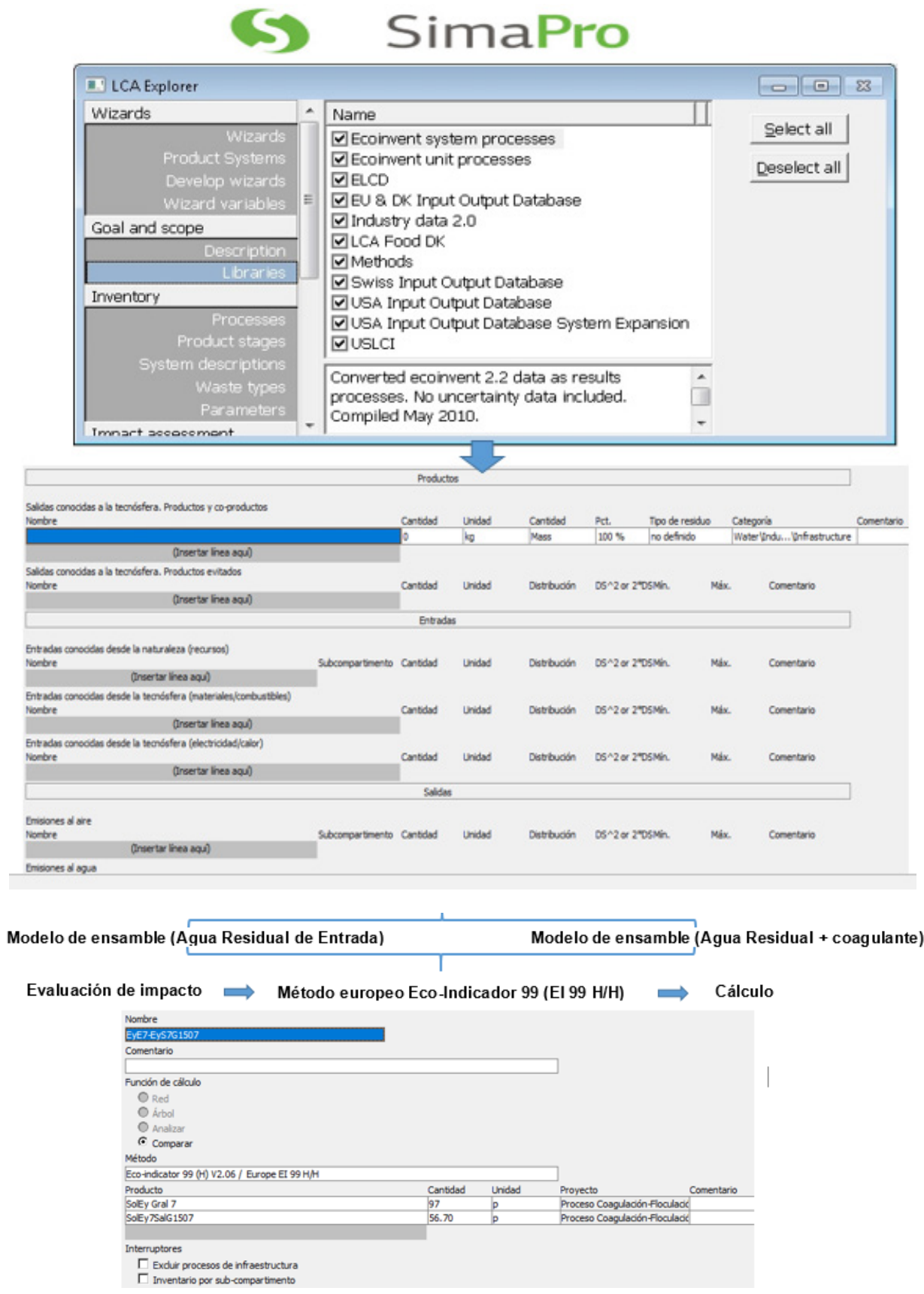

Figura 1. Esquema general del ACV de procesos industriales con el programa SimaPro. Figure 1. ACV general scheme of industrial processes with the SimaPro program. 
No obstante, se observó que una pequeña variación en los parámetros del proceso como es la dosis de coagulante, la velocidad de agitación y la temperatura pueden afectar de forma significativa la eficiencia del proceso de coagulación-floculación, mostrando solo el $10 \%$ de la reducción de la turbidez en aguas brutas de 90 NTU con el coagulante Ecotan Bio G-150 teniendo claro el efecto presente de la dosis de coagulante, la velocidad de agitación y la temperatura de trabajo. Por otro lado, los coagulantes Ecotan Bio 100 y 90D resultan ser afectados en el proceso por la velocidad de agitación y la temperatura, teniendo porcentajes de reducción de la turbidez de 12 y $24 \%$ respectivamente, para aguas brutas de 40 NTU (ver Tabla 2).

Tabla 2. Extracto de resultados del proceso fisicoquímico. Table 2. Results extract of the physicochemical process.

\begin{tabular}{lcccc}
\hline Coagulante & NTU inicial & Dosis $(\mathbf{m L})$ & $\mathbf{E}_{\text {entrada }}(\mathbf{k W h})$ & \% Reducción \\
\hline Bio 90D & 40 & 1 & 1254.73 & 84 \\
\hline Bio 100 & 40 & 1 & 2508.53 & 73 \\
\hline Bio G-150 & 40 & 6 & 2508.73 & 46 \\
\hline Bio 90D & 90 & 3.5 & 7526.52 & 80 \\
\hline Bio 100 & 90 & 3.5 & 3010.61 & 78 \\
\hline Bio G-150 & 90 & 3.5 & 3010.61 & 93 \\
\hline Bio 90D & 140 & 1 & 10034.14 & 50 \\
\hline Bio 100 & 140 & 6 & 1254.53 & 53 \\
\hline Bio G-150 & 140 & 1 & 10034.14 & 14 \\
\hline
\end{tabular}

En efecto, la capacidad de eliminación de la turbidez que pueden conseguir los coagulantes naturales en aguas residuales dentro del proceso de coagulación-floculación dependerá también del origen de las mismas, por ejemplo, en aguas residuales complejas es necesario el empleo de un método de tratamiento integral. El estudio realizado por Ponce y colaboradores muestra la necesidad de un tratamiento integral (Tecnología de Oxidación Avanzada + coagulaciónfloculación) para aumentar la eficiencia de los coagulantes de la serie Ecotan Bio en la reducción de la turbidez de hasta un $64 \%$ en aguas residuales del corcho (Ponce et al., 2017).

La investigación de Barrado (2016), muestra la eficiencia del proceso coagulación-floculación con el uso de dos coagulantes naturales (Acquapol C1 y S5T; Tanfloc) que poseen las mismas características del coagulante Ecotan Bio en la eliminación de microalgas (Chlorella, Microcystis, Oocystis y Scenedesmus) (Barrado, 2016), obteniendo el 90 $\%$ de eliminación de microalgas cuando el pH del agua residual es ajustado a $\mathrm{pH} 7$ y, con un $\mathrm{pH}$ entre 8 y 9 la eficiencia del proceso tiende a decrecer (20 a $40 \%$ de eliminación de microalgas), también se observó un efecto significativo en la eficiencia del proceso con mínimas variaciones de la dosis de coagulante, la velocidad y el tiempo de agitación, tal como se ha observado en la presente investigación.

\section{Análisis del Ciclo de Vida del proceso de coagulación- floculación con coagulantes naturales}

Se analizaron cuatro puntos de impacto ambiental relacionados con el efecto de cambio climático, la acidifi- cación/eutrofización, el suelo por el uso de la biomasa del co-producto del proceso y los recursos fósiles y minerales extraídos, los que fueron determinados con el programa SimaPro por medio de un análisis comparativo entre los tres coagulantes estudiados para los valores de turbidez de entrada de 40, 90, 131 y 140 NTU (Figura 2).

En la Figura 2 se observa que los efectos derivados por el uso de los coagulantes Ecotan Bio 90D y 100 en el proceso son prácticamente nulos sin llegar a superar valores de 1 milipunto, la generación de residuo en forma de lodo resultante del tratamiento es mínima. No obstante, el coagulante Ecotan Bio G-150 presenta efectos significativos en los cuatro puntos de interés, siendo los resultados más relevantes, los mostrados para aguas brutas tratadas con turbidez de 90 NTU, donde se aprecia un aporte de 0.75 milipuntos relacionado con el cambio climático, en este caso en la producción de $\mathrm{CO}_{2}$ generado por el consumo de energía durante el proceso de tratamiento y para la extracción de materia prima; en el apartado de eutrofización debido a la generación de lodos representa un aporte de 0.25 milipuntos; el aporte a favor de la generación de abono orgánico a partir del reciclaje de lodos es de 0.5 milipuntos, finalmente valores de hasta 7 milipuntos son generados por la extracción de recursos fósiles y minerales para la obtención de energía y coagulantes naturales.

También es de resaltar, que de acuerdo a los resultados de eficiencia en la remoción de la turbidez y los valores presentados en la Figura 2 para el Ecotan Bio G-150 en el proceso de coagulación-floculación, se puede establecer que la viabilidad de uso del coagulante Ecotan Bio G-150 no está del todo restringida siempre y cuando se ajuste la dosis óptima de coagulante y la energía de entrada, para disminuir de manera considerable los impactos en cuanto a la generación de lodos, el uso excesivo de coagulante, teniendo la posibilidad de establecer una economía sostenible para las empresas encargadas en el tratamiento de aguas residuales.

Los impactos generados por efecto de la eutrofización que se deriva de la producción de lodos para cada tipo de coagulante, no son de gran impacto, ya que se trata de coagulantes de origen natural los cuales presentan propiedades antimicrobianas para reducir la población microbiana de patógenos (www.servyeco.com).

Además, los estudios realizados principalmente del coagulante Ecotan Bio 90D frente al cloruro férrico en el proceso de tratamiento primario de aguas residuales urbanas e industrial, indican que Ecotan Bio 90D no presenta ecotoxicidad en un estudio in vivo en Vidrio fischeri, y presenta una elevada biodegradabilidad en condiciones anaeróbicas y aeróbicas (Sabia et al., 2014).

Los resultados obtenidos en el ACV del proceso de coagulación-floculación con los diferentes coagulantes ensayados, muestran la viabilidad de implementación del proceso en las diferentes industrias encargadas en el tratamiento de aguas residuales. Los índices de impacto generados en el programa SimaPro no indican un efecto negativo al ambiente, siempre y cuando se establezcan las condiciones 


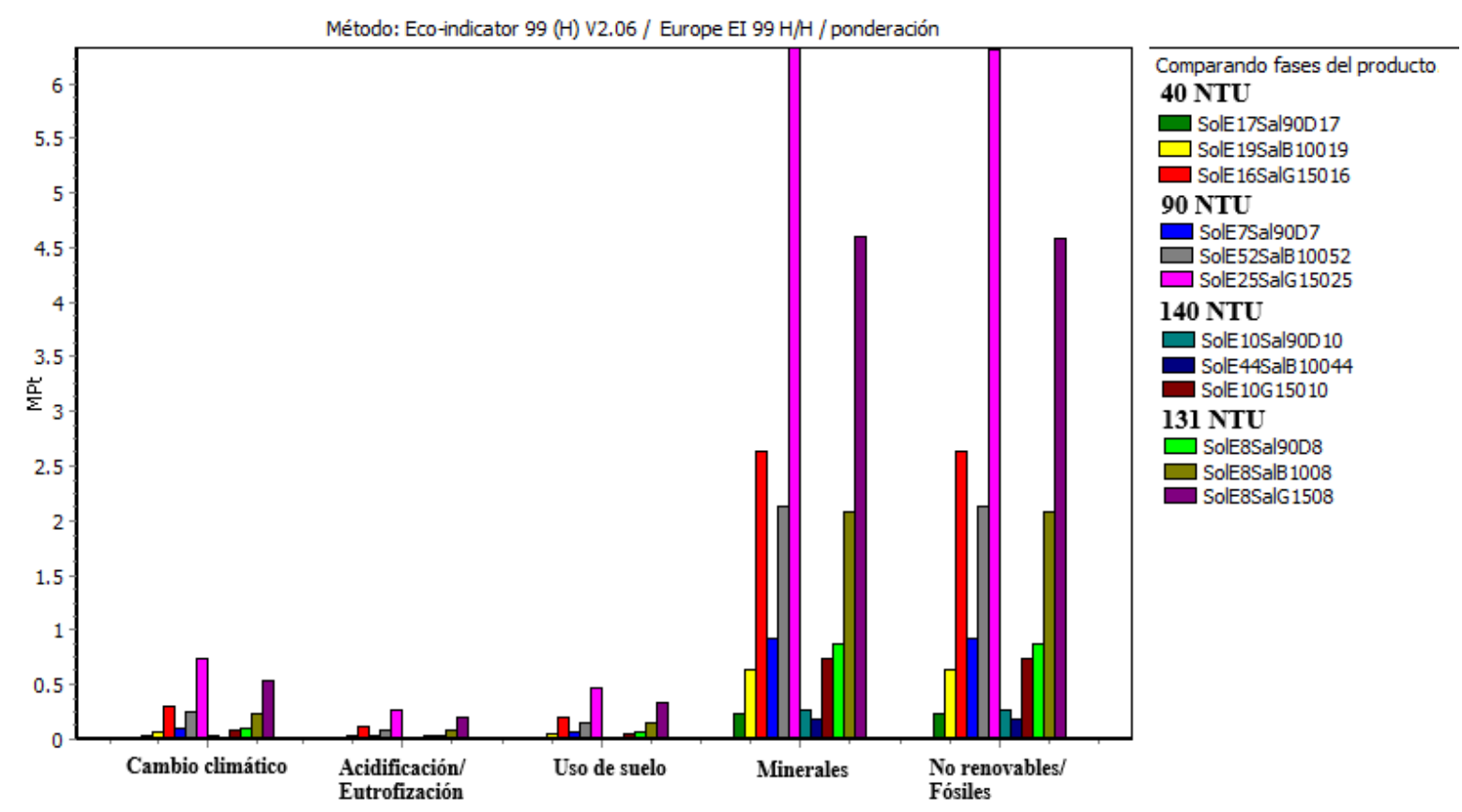

Mpt: milipuntos; Sal90D: Ecotan Bio 90D; SalB100: Ecotan Bio 100; SalG150: Ecotan Bio G-150

Figura 2. Impacto ambiental en repuesta al tratamiento de aguas residuales con un proceso físico-químico + coagulante natural para una turbidez de entrada de 40, 90 y 140 NTU para el Ensayo de entrenamiento y 131 NTU para el Ensayo de testeo.

Figure 2. Environmental impact in response to wastewater treatment with a physical-chemical process + natural coagulant for an input turbidity of 40,90 and 140 NTU for the Training Assay and 131 NTU for the Testing Assay.

adecuadas en el proceso de coagulación-floculación. Esto permite una economía sostenible que minimice la extracción de recursos naturales, implemente el uso de las $3 R$ en caso de generación de residuos (lodos) y el reúso del recurso agua.

\section{CONCLUSIÓN}

El ajuste óptimo de los parámetros necesarios en el proceso de coagulación-floculación es primordial para la optimización del proceso en el tratamiento de las aguas residuales, así como, para reducir el impacto ambiental por el abuso de reactivos químicos y energía, de acuerdo a los resultados obtenidos en esta investigación.

El ACV del proceso de coagulación-floculación con los diferentes coagulantes naturales analizados muestra la viabilidad del proceso para ser implementado en las plantas de tratamiento de aguas residuales, ya que los impactos ambientales generados por el programa SimaPro en los cuatro puntos de estudio no presentan efectos negativos al ambiente, siempre que se establezca las condiciones adecuadas del proceso de tratamiento de aguas residuales.

\section{REFERENCIAS}

Barrado, M. 2016. Eliminación de microalgas de las aguas mediante métodos físico-químicos. Tesis Doctoral. Universidad de Extremadura, Badajoz, España.

Dorca, J., Teresinha, M., Moreno, S., da Cunha, G. y FagundesKlen, M. 2017. Evaluation of the combined process of coagulation/flocculation and microfiltration of Cassava
Starch wastewater: removal efficiency and membrane fouling. Water Air Soil Pollut. 228(238): 1-12.

Heijungs, R., Huppes, G. y Guinée, J. 2010. Life cycle assessment and sustainability analysis of products, materials and technologies. Toward a scientific framework for sustainability life cycle analysis. Polymer Degradation and Stability. 95: 422-428.

Höjer, M., Ahlroth, S., Dreborg, K., Ekvall, T., Finnveden, G., Hjelm, O., Hochschorner, E., Nilsson, M. y Palm, V. 2008. Scenarios in selected tools for environmental systems analysis. Journal of Cleaner Production. 16: 1958-1970.

Ficha técnica de coagulantes naturales de la serie ECOTAN BIO. [Consultado 14 de febrero de 2018]. Disponible en: http:// www.servyeco.com/coagulantes-naturales.html

loannou, L., Puma, G. y Fatta, D. 2015. Treatment of winery wastewater by physicochemical, biological and advanced processes: A review. Journal of Hazardous Materials. 286: 343-368.

Reporte mensual de las características físico-químicas de las aguas residuales de la Estación Depuradora de Aguas Residuales de Logroño (portal web La Rioja), La Rioja, España. [Consultado enero de 2017]. Disponible en: http://www. larioja.org/consorcio-aguas/es/depuracion/instalaciones/ depuradoras-servicio/estaciones-depuradoras-aguasresiduales

Lorenzo, Y., Alfonsín, C., Amores, M., Aldea, X., Marin, D., Moreira, M. y Feijoo, G. 2016a. Beyond the conventional life cycle inventory in wastewater treatment plants. Science of the Total Environment. 553: 71-82.

Lorenzo, Y., Vázquez, I., Amores, M., Termes, M., Marín, D., Moreira, M. y Feijoo, G. 2016b. Benchmarking wastewater treatment 
plants under an eco-efficiency perspective. Science of the Total Environment. 566-567: 468-479.

Lorenzo, Y., Vázquez, I., Chenel, S., Marín, D., Moreira, M. y Feijoo, G. 2015. Eco-efficiency analysis of Spanish WWTPs using the LCA+DEA method. Water Research. 68: 651-666.

Manuilova, A., Suebsiri, J. y Wilson, M. 2009. Should life cycle assessment be part of the environmental impact assessment? Case study: EIA of $\mathrm{CO}_{2}$ capture and storage in Canada. Energy Procedia. 1: 4511-4518.

Mateus, G., Formentini, D., Nishi, L., Fagundes, M., Gomes, R. y Bergamasco, R. 2017. Coagulation/flocculation with Moringa Oleifera and membrane filtration for dairy wastewater treatment. Water Air Soil Pollut. 228(342): 1-13.

Oladoja, N. 2015. Headway on natural polymeric coagulants in water and wastewater treatment operations. Journal of Water Process Engineering. 6: 174-192.

Ponce, L., Miralles, S., Oller, I., Agüera, A., Trinidad, M., Yuste, F. y Malato, S. 2017. Cork boiling wastewater treatment and reuse through combination of advanced oxidation technologies. Environmental Science and Pollution Research. 24: 63176328.

Rebah, F. y Siddeeg. 2017. Cactus an eco-friendly material for wastewater treatment: A review. Journal of Materials and Environmental Sciences. 8(5): 1770-1782.

Roche, T. 2004. The design for environmental compliance workbench tool. In: Talaba D., Roche T. Product Engineering. Springer, Dordrecht (ed.). 3-16 pp.
Rodriguez, G., Molinos, M., Hospido, A., Hernández, F., Moreira, M. y Feijoo, G. 2011. Environmental and economic profile of six typologies of wastewater treatment plants. Water Research. 45: 5997-6010.

Sabia, G., Giuliano, A. y Farina, R. 2014. Assessment of the ecotoxicological effects related to the use of natural organic coagulants in the primary treatment of wastewaters at urban and industrial level. Istituto Superiore per la Protezione e la Ricerca Ambientale, Roma, Italia.

Saritha, V., Srinivas, N. y Srikanth, N. 2017. Analysis and optimization of coagulation and flocculation process. Appl. Water Sci. 7: 451-460.

Seow, Y., Goffin, N., Rahimifard, S. y Woolley, E. 2016. A 'design for energy minimization' approach to reduce energy consumption during the manufacturing phase. Energy. 109: 894-905.

Singh, A., Kamble, S., Sawant, M., Chakravarthy, Y., Kazmi, A., Aymerich, E., Starkl, M., Ghangrekar, M. y Philip, L. 2016. Technical, hygiene, economic, and life cycle assessment of full-scale moving bed biofilm reactors for wastewater treatment in India. Environ. Sci. Pollut. Res. 25: 2552-2569.

Yanguas, J. 2017. Optimización en el proceso de depuración de aguas residuales urbanas. Trabajo Fin de Grado. Universidad de La Rioja, Logroño, España. 